\title{
An Efficient Numerical Method for a Class of Nonlinear Volterra Integro-Differential Equations
}

\author{
M. H. Daliri Birjandi (iD, J. Saberi-Nadjafi $(\mathbb{D}$, and A. Ghorbani \\ Department of Applied Mathematics, School of Mathematical Sciences, Ferdowsi University of Mashhad, Mashhad, Iran \\ Correspondence should be addressed to J. Saberi-Nadjafi; najafil41@gmail.com
}

Received 6 April 2018; Revised 8 June 2018; Accepted 13 June 2018; Published 1 August 2018

Academic Editor: Mehmet Sezer

Copyright ( 2018 M. H. Daliri Birjandi et al. This is an open access article distributed under the Creative Commons Attribution License, which permits unrestricted use, distribution, and reproduction in any medium, provided the original work is properly cited.

\begin{abstract}
We investigate an efficient numerical method for solving a class of nonlinear Volterra integro-differential equations, which is a combination of the parametric iteration method and the spectral collocation method. The implementation of the modified method is demonstrated by solving several nonlinear Volterra integro-differential equations. The results reveal that the developed method is easy to implement and avoids the additional computational work. Furthermore, the method is a promising approximate tool to solve this class of nonlinear equations and provides us with a convenient way to control and modify the convergence rate of the solution.
\end{abstract}

\section{Introduction}

Many physical phenomena in different fields of sciences and engineering have been formulated using integro-differential equations. The nonlinear integro-differential equations play a crucial role to describe many process like fluid dynamics, biological models and chemical kinetics, population, potential theory, polymer theology, and drop wise condensation (see [1-4] and the references cited therein). In fact analytical solutions of integro-differential equations either do not exist or they are hard to compute. Eventually an exact solution is computable, the required calculations may be tedious, or the resulting solution may be difficult to interpret. Due to this, it is required to obtain an efficient numerical solution. In literature there exist several numerical methods for solving integro-differential equations such as successive approximation method, meshless method [5], Taylor polynomial [6], Tau method [4], wavelet-Galerkin method [7], Adomain decomposition method [8], Homotopy perturbation method [9], Homotopy analysis method [10], Sinc collocation [11], Legendre polynomials [12], and Taylor collocation method [13]. The monograph by Bruner [14] includes a wealth of material on the theory and numerical methods for Volterra integro-differential equations.

The parametric iteration method (PIM) is an analytic approximate method that provides the solution of linear and nonlinear problem as a sequence of iterations. In fact, the PIM as a fixed- point iteration method is a reconstruction of variational iteration method [15]. The PIM, however, suffers from a number of restrictive measures, such as the resulting integrals in its iterative relation which may not be performed analytically. Also, the implementation of the PIM generally leads to calculation of unneeded terms, in which more time is consumed in repeated calculations for series solutions.

In order to overcome these shortcomings, a useful improvement of the PIM was proposed in [16]. Therefore, the strategy that will be pursued in this work rests mainly on establishing a simple algorithm, requiring no tedious computational work, based on the improved PIM and the spectral collocation technique for obtaining an accurate solution for the following nonlinear Volterra integro-differential equation (VIDE):

$$
\begin{aligned}
& u^{\prime}(t)=f(t)+\int_{0}^{t} k(t, s) G(u(s)) d s, \quad t \in[0, T] \\
& u(0)=u_{0},
\end{aligned}
$$

where the kernels $k(t, s), f(t)$ and $G(u(s))$ are smooth functions. The existence and uniqueness of the solution for (1) are presented in [17].

To demonstrate the utility of the proposed method, some examples of the nonlinear VIDEs are given, which are 
solved using the established method. The obtained results are compared with the numerical solutions. In all cases, the present algorithm performed excellently.

\section{The Basic Idea of the PIM}

The PIM gives a rapidly convergent approach by using successive approximations of the exact solution if such a solution exists; otherwise the approximations can be used for numerical purposes. The idea of the PIM is very simple and straightforward. To explain the PIM, consider (1) as below:

$$
L[u(t)]+N[u(t)]=f(t),
$$

where $L$ with the property $L v \equiv 0$ when $v \equiv 0$ and it denotes the auxiliary linear operator with respect to $u$. In (2) $N$ is a nonlinear continuous operator with respect to $u$ and $f(t)$ is the source term.

According to $[15,16]$, we construct the following family of the explicit PIM for (2) as

$$
L\left[u_{k+1}(t)-u_{k}(t)\right]=h H(t) A\left[u_{k}(t)\right],
$$

where

$$
\begin{aligned}
A\left[u_{k}(t)\right] & =L\left[u_{k}(t)\right]+N\left[u_{k}(t)\right]-f(t) \\
& =u_{k}^{\prime}(t)-\int_{0}^{t} k(t, s) G\left(u_{k}(s)\right) d s-f(t),
\end{aligned}
$$

with the initial condition

$$
u_{k+1}(0)=u_{0}
$$

Also we can construct a family of the implicit PIM for (2) as follows:

$$
\begin{aligned}
L & {\left[u_{k+1}(t)-u_{k}(t)\right] } \\
& =h H(t)\left\{L\left[u_{k}(t)\right]+N\left[u_{k+1}(t)\right]-f(t)\right\},
\end{aligned}
$$

with the above initial condition.

$u_{0}(t)$ is the initial guess which can be freely found from solving its corresponding linear equation $\left(L\left[u_{0}(t)\right]=0\right.$ or $\left.L\left[u_{0}(t)\right]=f(t)\right)$ and the subscript $k$ denotes the $k$ th iteration. Accordingly the approximations of $u_{k}(t), k \geq 0$ for the PIM iterative relation will be obtained readily in the auxiliary parameter $h$. Consequently, the exact solution can be obtained by using

$$
u(t)=\lim _{k \longrightarrow \infty} u_{k}(t)
$$

The parametric iteration formula (3) makes a recurrence sequence $u_{k}(t)$. Obviously, the limit of the sequence will be the solution of (1) if the sequence is convergent. In the following, we give a proof of convergence of the PIM. Here we assume that for every $k, u_{k} \in C^{1}[0, T]$ and $\left\{u_{k}^{\prime}\right\}$ is uniformly convergent.

Theorem 1. If the sequence $u_{k}(t)$ converges, where $u_{k}(t)$ is produced by the parametric iteration formulation of (3), then it must be the exact solution of (1).
Proof. If the sequence $\left\{u_{k}(t)\right\}$ converges, we define

$$
U(t)=\lim _{k \longrightarrow \infty} u_{k}(t),
$$

and it holds

$$
U(t)=\lim _{k \longrightarrow \infty} u_{k+1}(t)
$$

From (16) and (9) and the definition of $L$, we can easily acquire

$$
\begin{aligned}
& \lim _{k \rightarrow \infty} L\left[u_{k+1}(t)-u_{k}(t)\right]=L \lim _{k \rightarrow \infty}\left[u_{k+1}(t)-u_{k}(t)\right] \\
& \quad=0
\end{aligned}
$$

From (10) and according to (3), we obtain

$$
h H(t) \lim _{k \rightarrow \infty} A\left[u_{k}(t)\right]=L \lim _{k \longrightarrow \infty}\left[u_{k+1}(t)-u_{k}(t)\right]=0 .
$$

Since $h \neq 0$ and also $H(t) \neq 0$ for all $t$, the relation (11) gives us

$$
\lim _{k \rightarrow \infty} A\left[u_{k}(t)\right]=0 .
$$

From (12) and the continuity property of the operator $G$, it follows that

$$
\begin{aligned}
\lim _{k \rightarrow \infty} A\left[u_{k}(t)\right] & \\
= & \lim _{k \rightarrow \infty}\left(u_{k}^{\prime}(t)-\int_{0}^{t} k(t, s) G\left(u_{k}(s)\right) d s-f(t)\right) \\
= & \left(\lim _{k \longrightarrow \infty} u_{k}(t)\right)^{\prime}-\int_{0}^{t} k(t, s) G\left(\lim _{k \longrightarrow \infty} u_{k}(s)\right) d s \\
& \quad-f(t)=U^{\prime}(t)-\int_{0}^{t} k(t, s) G(U(s)) d s-f(t) .
\end{aligned}
$$

From (12) and (13), we get

$$
U^{\prime}(t)-\int_{0}^{t} k(t, s) G(U(s)) d s-f(t)=0,
$$

$$
0 \leq s, t \leq T \text {. }
$$

On the other hand, in view of the initial condition of the $(k+$ 1)th order PIM and (9), it holds that

$$
U(0)=\lim _{k \longrightarrow \infty} u_{k+1}(0)=u(0)=u_{0} .
$$

Hence, according to the expressions (14) and (15), $U(t)$ must be the exact solution of (1) and this ends the proof.

It is obvious that the convergence of the sequence (16) depends upon the initial guess $u_{0}(t)$, the auxiliary linear operator $L$, the auxiliary parameter $h$, and the auxiliary function $H(t)$. Fortunately, the PIM provides us with the great freedom of choosing these items. Thus, as long as $u_{0}(t), L$, $h$, and $H(t)$ are property chosen so that the sequence (16) converges in a region $0 \leq t \leq T$, it should converge to the exact solution in this region. Therefore, the combination of the convergence theorem and the freedom of the choice of the above factors establishes the cornerstone of the validity and flexibility of the PIM. 
Remark 2. In the case of failure of convergence of the PIM, the presence of the parameter $h$ in (3) or (6) could play a very important role in the frame of the PIM. Although we can find a valid region of $h$ for every physical problem by plotting the solution or its derivatives versus the parameter $h$ in some points, an approximate optimal value of the convergence accelerating parameter $h$ can be determined at the order of approximation by the residual error [15]

$$
\begin{aligned}
\operatorname{Res} & (h) \\
= & \int_{0}^{T}\left\{L\left[u_{k}(t ; h)\right]+N\left[u_{k}(t ; h)\right]-f(t)\right\}^{2} d x .
\end{aligned}
$$

One can minimize (16) by imposing the requirement $d \operatorname{Res}(h) / d h=0$.

\section{A Spectral Collocation PIM}

In general, the application of the PIM to solve the nonlinear VIDEs leads to the calculation of unneeded and repeated terms. The unneeded and repeated calculations may or may not lead to faster convergence. Also, since the PIM provides the solution as a sequence of iterates, its successive iterations may be very complex so that the resulting integrals in its iterative relation may not be performed analytically. In this section, we will overcome this shortcoming of the original PIM for solving (1) by suggesting a spectral collocation PIM. As will be shown in this paper later, the proposed method will be very simple to implement and save time and calculations.

Consider the basis functions $\phi_{j}$ which are polynomials of degree $N-1$ satisfying $\phi_{j}\left(t_{k}\right)=\delta_{j, k}$ for the shifted Chebyshev nodes (note that $t_{1}=T$ and $t_{N}=0$ )

$$
t_{k}=\frac{T}{2}\left[\cos \left(\frac{(k-1) \pi}{N-1}\right)+1\right], \quad k=1, \ldots, N .
$$

The unknown function $u(t)$ is approximated as a truncated series of polynomials. The polynomial

$$
p(t) \cong u(t)=\sum_{j=1}^{N} u_{j} \phi_{j}(t)
$$

interpolates the points $\left(t_{j}, u_{j}\right), j=1, \ldots, N$; that is, $p(\mathbf{t})=\mathbf{u}$, where $\mathbf{t}=\left(t_{1}, \ldots, t_{N}\right)$ and $\mathbf{u}=\left(u_{1}, \ldots, u_{N}\right)$. The values of the interpolating polynomial's first derivative at the nodes are $p^{\prime}(\mathbf{t})=D^{(1)} \mathbf{u}$, and the value of integral at the nodes is defined by $\int_{0}^{t} k(t, s) u(s) d s=V \cdot \mathbf{u}$, where $V$ is the Volterra integration matrix $[18,19]$.

Generally, in order to solve problem (1) using a spectral collocation scheme, the interpolating polynomial $p(t)$ is required to satisfy the equation at the interior nodes. The values of the interpolating polynomial at the interior nodes $t_{2}, \ldots, t_{N}$ are $p\left(\mathbf{t}_{m}\right)=(\mathbf{u})_{m}=I_{m,:} \mathbf{u}(m=1: N-1)$ and the derivative value is $p^{\prime}\left(\mathbf{t}_{m}\right)=D_{m,:}^{(1)} \mathbf{u}$. The initial condition that involves the interpolating polynomial can be handled by using the formula $p\left(\mathbf{t}_{N}\right)=(\mathbf{u})_{N}=I_{N,:} \mathbf{u}$, where $I_{N, \text { : }}$ denotes the last row of the $(N \times N)$ identity matrix.
For the interpolating polynomial to satisfy the nonlinear VIDE of (1) at each interior node, the collocation equation

$$
\begin{aligned}
& p^{\prime}\left(\mathbf{t}_{m}\right)=f\left(\mathbf{t}_{m}\right)+\int_{0}^{\mathbf{t}} k(\mathbf{t}, s) G(p(s)) d s, \\
& p\left(\mathbf{t}_{N}\right)=u_{0},
\end{aligned}
$$

should be satisfied. Substituting the differentiation and integration matrix relations into equation (19), we get

$$
\left[\begin{array}{c}
D_{m,:}^{(1)} \\
I_{N,:}
\end{array}\right] \mathbf{u}=\left[\begin{array}{l}
\mathbf{f}_{m} \\
u_{0}
\end{array}\right]+\left[\begin{array}{c}
I_{m,:}(V \cdot G(\mathbf{u})) \\
0
\end{array}\right],
$$

where $\mathbf{f}_{m}=\left\{f\left(t_{1}\right), \ldots, f\left(t_{N-1}\right)\right\}$. Now, in view of (3) and the definitions of $L$ and $A$, by substituting the differentiation and integration matrix relations, we will have the following explicit PIM for solving (1) which is called the spectral PIM (SPIM):

$$
\begin{aligned}
& \mathbf{u}_{k+1}=\mathbf{u}_{k}+h\left[\begin{array}{c}
D_{m,:}^{(1)} \\
I_{N,:}
\end{array}\right]^{-1} \\
& \cdot\left(\left[\begin{array}{c}
D_{m,:}^{(1)} \\
I_{N,:}
\end{array}\right] \mathbf{u}_{k}-\left[\begin{array}{c}
\mathbf{f}_{m} \\
u_{0}
\end{array}\right]-\left[\begin{array}{c}
I_{m,:}\left(V \cdot G\left(\mathbf{u}_{k}\right)\right) \\
0
\end{array}\right]\right),
\end{aligned}
$$

where for simplicity we chose $H(t) \equiv 1$. If we define $\mathbf{L}=$ $\left[D_{m,:}^{1}, I_{N,:}\right]^{T}, \mathbf{f}=\left[\mathbf{f}_{m}, u_{0}\right]^{T}$, and $\mathbf{N u}_{k}=\left[I_{m,:}\left(V \cdot G\left(\mathbf{u}_{k}\right)\right), 0\right]^{T}$, then we will have the following explicit iterative relation for finding the solution vector $\mathbf{u}_{k+1}$ :

$$
\mathbf{u}_{k+1}=\mathbf{u}_{k}+h \mathbf{L}^{-1}\left(\mathbf{L} \mathbf{u}_{k}-\mathbf{f}-\mathbf{N u}_{k}\right)
$$

Here the vector $\mathbf{u}_{k+1}$ is defined as

$$
\mathbf{u}_{k+1}=\left\{u_{k+1}\left(t_{1}\right), \ldots, u_{k+1}\left(t_{N-1}\right)\right\} .
$$

In using the SPIM algorithm above, we begin by choosing the best possible initial approximation that satisfies the initial condition. To this end, we may determine the initial approximation by solving $\mathbf{L u}_{0}=0$ or $\mathbf{L} \mathbf{u}_{0}=\mathbf{f}$. Thus, starting from the initial approximation $\mathbf{u}_{0}(t)$, we can use the recurrence formula (22) to successively obtain directly $\mathbf{u}_{k+1}(t)$ for $k \geq 0$.

\section{Test Problems}

In this section, we demonstrate the effectiveness of the SPIM by applying the method to three nonlinear NVIDs. All of the numerical computations have been performed in MATLAB R2014a and terminated when the current iterate satisfies $\| \mathbf{u}_{k}-$ $\mathbf{u}_{k-1} \| \leq 10^{-16}$, where $\mathbf{u}_{k}$ is the solution vector of the $k$ th SPIM iteration.

Example 1. Consider the following nonlinear VIDE [20]:

$$
u^{\prime}(t)=\frac{1}{\varepsilon}\left(u-u^{2}-u \int_{0}^{t} u(s) d s\right), \quad t \in[0,1]
$$




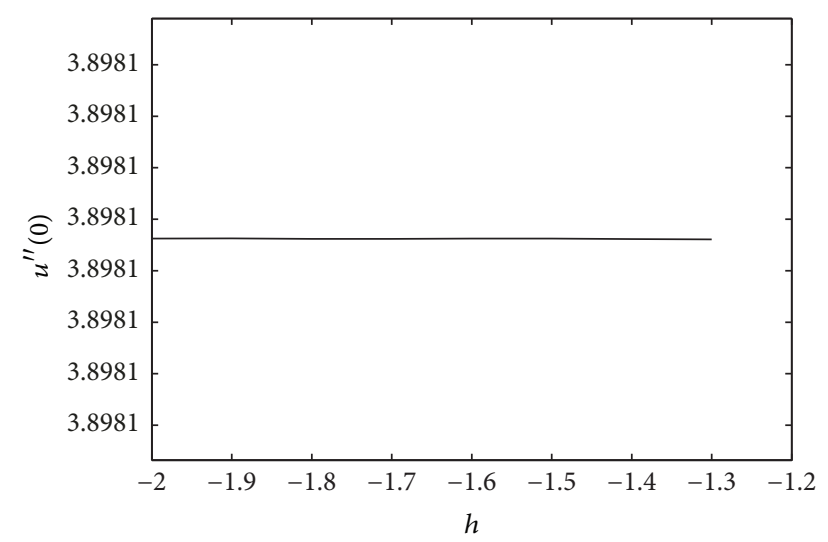

FIgure 1: The valid region of $h$ for the explicit spectral PIM when $N=15$ for Example 1 .

with the initial condition $u(0)=0.1$. Here we aim to solve the above Volterra population equation for the value $\varepsilon=1 / 10$. To use the proposed method in this paper, i.e., (22), we could choose

$$
\begin{aligned}
L[u(t)] & =u^{\prime}(t), \\
N[u(t)] & =\frac{1}{\varepsilon}\left(u-u^{2}-u \int_{0}^{t} u(s) d s\right), \\
g(t) & \equiv 0 .
\end{aligned}
$$

To investigate the valid region $h$ of the solution obtained via the explicit spectral PIM algorithm (22) for $N=15$ of (24) with $\varepsilon=1 / 10$, we try to plot the curve of $u^{\prime \prime}(0)$ with respect to $h$, as shown in Figure 1 . According to this curve, it is easy to discover the valid region of $h$. It is usually convenient to investigate the valid region of $h$ for the PIM by means of such kinds of the curves.

According to Figure 1, it could be seen that the explicit spectral PIM for $h=-1$ and $N=15$ (even for large $N$ ) is not a convergent approach for solving (24). The presence of the auxiliary parameter $h$ in the framework of the explicit spectral PIM could play a very important role. As mentioned above, we can find an approximate optimal value for $h$ from (16) by estimating the residual error $\operatorname{Res}(h)$ in a sequence of values $h$, as the value of $h$ with the lowest residual will be the approximate optimal $h$. Figure 2 shows the approximate optimal value of $h$ for the explicit spectral PIM for $N=15$, i.e., $h=-1.47$ with two decimal digits.

Figure 3(b) shows the absolute error of the explicit spectral PIM for $N=15$ and $h=-1.47$. Also the behavior of the numerical and explicit spectral PIM solutions of this example for $N=15$ and $h=-1.47$ is presented in Figure 3(a).

Example 2. Consider the following nonlinear VIDE:

$$
u^{\prime}(t)=1-\frac{1}{2} t+\frac{1}{2} e^{-t^{2}}+\int_{0}^{t} t s e^{-u^{2}(s)} d s
$$

with initial condition $u(0)=0$ and the exact solution $u(t)=t$, [21].

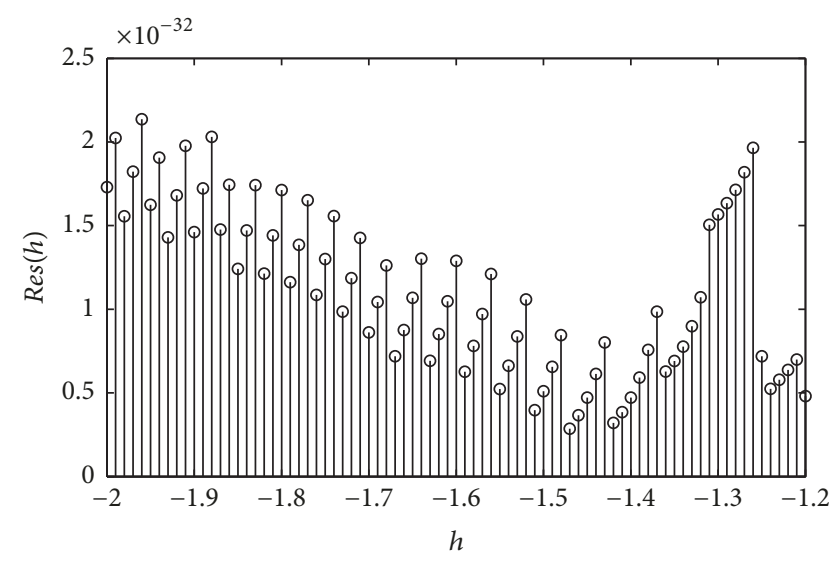

FIGURE 2: The approximate optimal $h(h=-1.47)$ for $N=15$ for Example 1.

To investigate the valid region $h$ for the solution obtained via the explicit spectral PIM algorithm (22) for $N=10$ of (26), here we plot the curve of $u^{\prime \prime}(0)$ with respect to $h$, as shown in Figure 4.

Figure 5 shows the approximate optimal value of $h$ of the explicit spectral PIM when $N=10$, i.e., $h=-1.1$ with one decimal digit.

Figure 6 shows the absolute error of the explicit spectral PIM for $N=10$ and $h=-1.1$.

Example 3. Consider the following nonlinear VIDE [22]:

$$
u^{\prime}(t)=f(t)+\int_{0}^{t}(t-s) \ln (1+u(s)) d s
$$

where

$$
\begin{aligned}
& f(t)=\frac{1}{24}\left(8+9 t^{2}+\frac{12}{\sqrt{1+t}}-8 \sqrt{1+t}\right. \\
& \left.-4 t(-6+5 \sqrt{1+t})-12 t^{2} \ln (1+\sqrt{1+t})\right)
\end{aligned}
$$

with the initial condition $u(0)=1$ and the corresponding exact solution is given by $u(t)=\sqrt{1+t}$.

To investigate the valid region $h$ of the solution obtained via the explicit spectral PIM algorithm (22) for $N=10$ of (27), here we plot the curve of $u^{\prime \prime}(0)$ with respect to $h$, as shown in Figure 7.

Figure 8 shows the approximate optimal value of $h$ for the explicit spectral PIM when $N=10$, i.e., $h=-0.8$ with one decimal digit.

Figure 9 shows the absolute error of the explicit spectral PIM for $N=10$ and $h=-0.8$.

\section{Conclusion}

In this paper, we presented a new application of the spectral parametric iteration method (PIM) for solving a class of nonlinear Volterra integro-differential equations (VIDEs).This new method is easy to implement and is accurate when 


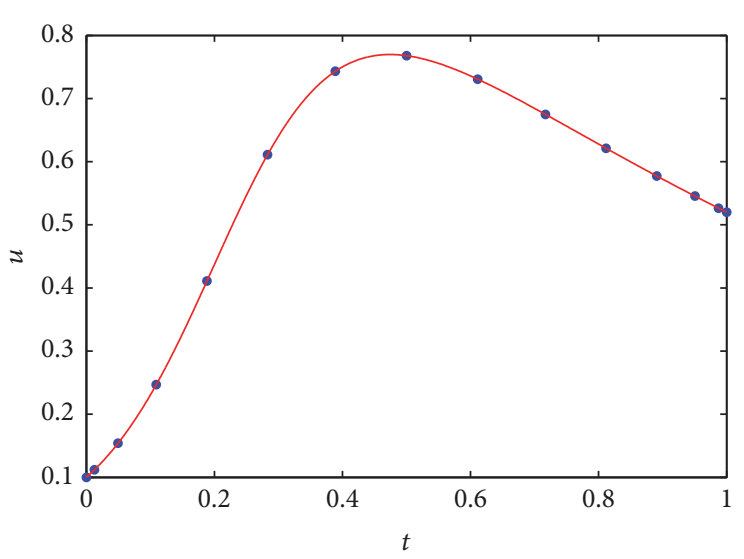

- Approximate solution

- Numerical solution

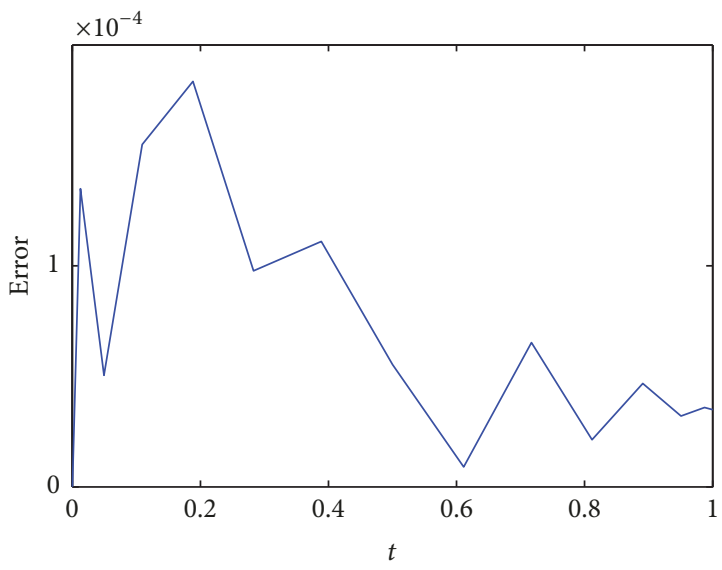

(a)

(b)

FIGURE 3: (a) Approximate solution of the explicit spectral PIM for $N=15$. (b) Absolute error of the explicit spectral PIM for $N=15$ and $h=-1.47$ for Example 1 .

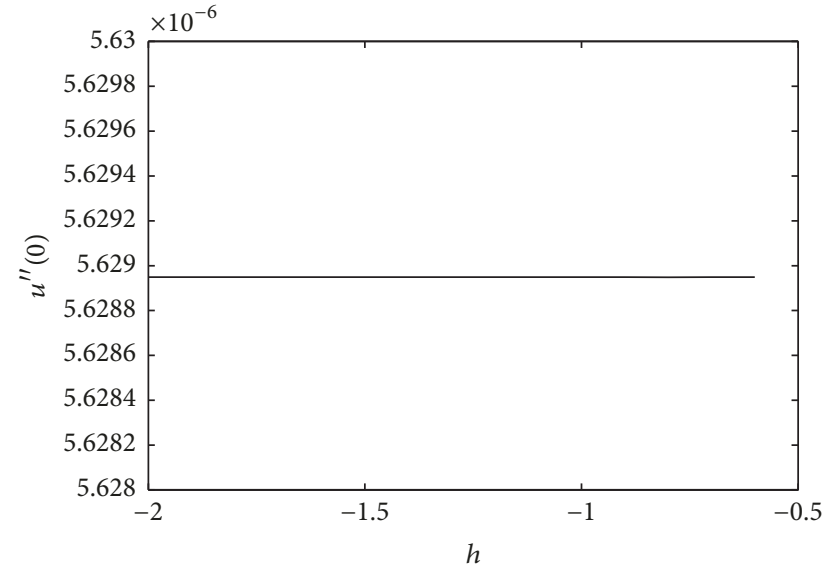

FIGURE 4: The valid region $h$ for the explicit spectral PIM when $N=$ 10 for Example 2.

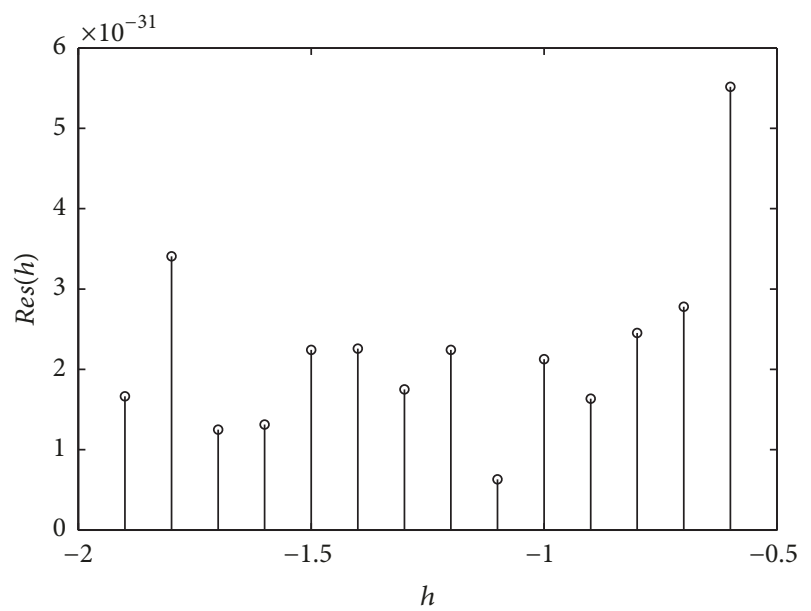

FIGURE 5: The approximate optimal $h(h=-1.1)$ for $N=10$ for Example 2.

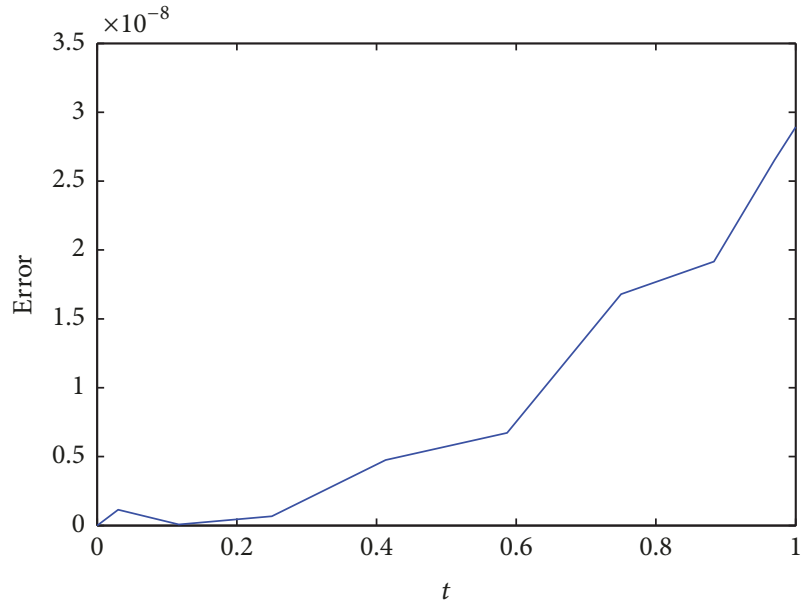

Figure 6: Absolute error of the explicit spectral PIM for $N=10$ and $h=-1.1$ for Example 2 .

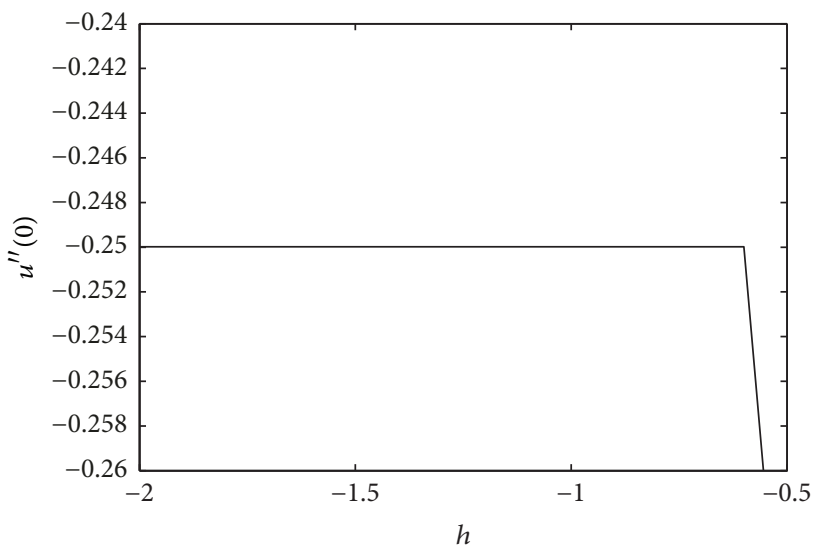

FIGURE 7: The valid region $h$ of the explicit spectral PIM when $N=$ 10 for Example 3. 


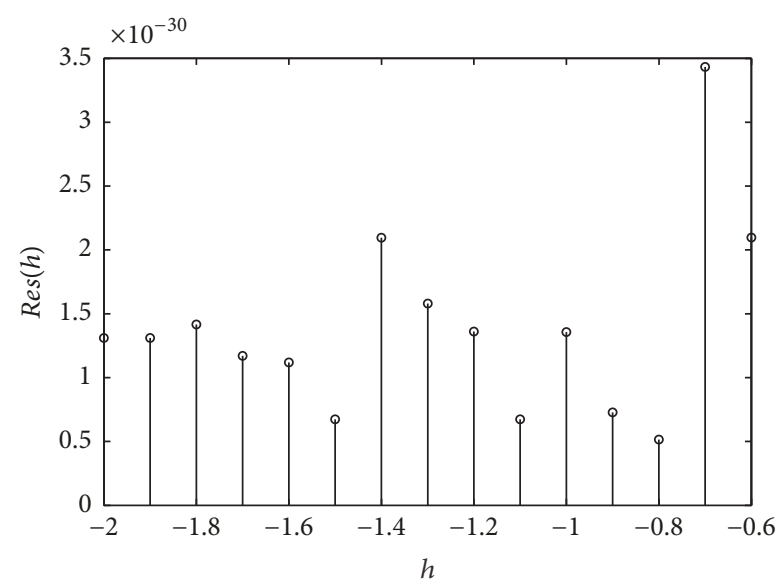

Figure 8: The approximate optimal $h(h=-0.8)$ for $N=10$ for Example 3.

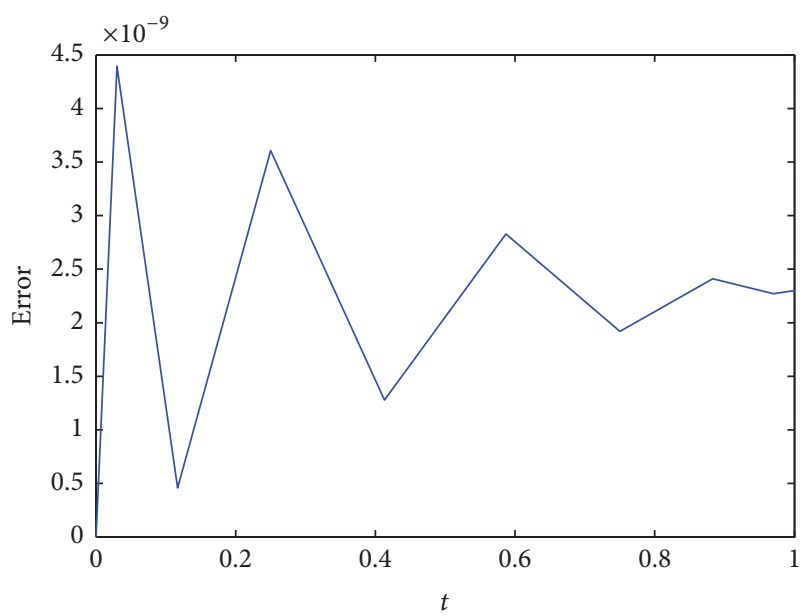

FIGURE 9: Absolute error of the explicit spectral PIM for $N=10$ and $h=-0.8$ for Example 3 .

applied to the nonlinear VIDEs. The numerical results of the spectral PIM were compared with the exact solutions and excellent agreement was obtained. This could confirm the validity of the proposed spectral PIM as a suitable method for solving this class of the nonlinear VIDEs.

\section{Data Availability}

The data used in our manuscript to support the findings of this study are included within the article. Furthermore, readers can access the data supporting the conclusions of the study by referring to [DOI or other persistent identifiers] in the references of our manuscript.

\section{Conflicts of Interest}

M. H. Daliri Birjandi, J. Saberi-Nadjafi, and A. Ghorbani declare that there are no conflicts of interest regarding the publication of this paper.

\section{References}

[1] I. Abdul, Introduction to Integral Equations with Application, Wiley, NewYork, NY, USA, 1999.

[2] S. Shaw and J. R. Whiteman, "Adaptive space-time finite element solution for Volterra equations arising in viscoelasticity problems," Journal of Computational and Applied Mathematics, vol. 125, no. 1-2, pp. 337-345, 2000.

[3] V. Volterra, Theory of Functionals And of Integral And IntegroDifferential Equations, Dover Publications, Inc., New York, NY, USA, 1959.

[4] A. J. Jerri, Introduction to Integral Equations with Applications, Marcel Dekker, New Yourk, NY, USA, 1999.

[5] M. Dehghan and R. Salehi, "The numerical solution of the non-linear integro-differential equations based on the meshless method," Journal of Computational and Applied Mathematics, vol. 236, no. 9, pp. 2367-2377, 2012.

[6] K. Maleknejad and Y. Mahmoudi, "Taylor polynomial solution of high-order nonlinear Volterra-Fredholm integro-differential equations," Applied Mathematics and Computation, vol. 145, no. 2-3, pp. 641-653, 2003.

[7] K. Maleknejad and F. Mirzaee, "Using rationalized Haar wavelet for solving linear integral equations," Applied Mathematics and Computation, vol. 160, no. 2, pp. 579-587, 2005.

[8] S. M. El-Sayed and M. R. Abdel-Aziz, "A comparison of Adomian's decomposition method and wavelet-Galerkin method for solving integro-differential equations," Applied Mathematics and Computation, vol. 136, no. 1, pp. 151-159, 2003.

[9] J.-H. He, "The homotopy perturbation method nonlinear oscillators with discontinuities," Applied Mathematics and Computation, vol. 151, no. 1, pp. 287-292, 2004.

[10] S. Behzadi, S. Abbasbandy, S. T. Allahviranloo, and A. Yildirim, "Application of homotopy analysis method for solving a class of nonlinear Volterra-Fredholm integro-differential equations," Journal of Applied Analysis and Computation, pp. 1-14, 2012.

[11] M. Zarebnia, "Sinc numerical solution for the Volterra integrodifferential equation," Communications in Nonlinear Science and Numerical Simulation, vol. 15, no. 3, pp. 700-706, 2010.

[12] E. Tohidi and O. R. N. Samadi, "Optimal control of nonlinear Volterra integral equations via Legendre polynomials," IMA Journal of Mathematical Control and Information, vol. 30, no. 1, pp. 67-83, 2013.

[13] A. Karamete and M. Sezer, "A Taylor collocation method for the solution of linear integro-differential equations," International Journal of Computer Mathematics, vol. 79, no. 9, pp. 987-1000, 2002.

[14] H. Brunner, Collocation Method for Volterra Integral and Related Functional Equations, Cambridge University Press, Cambridge, London, UK, 2004.

[15] M. H. Daliri Birjandi, J. Saberi-Nadjafi, and A. Ghorbani, "A novel method for solving nonlinear volterra integro-differential equation systems," Abstract and Applied Analysis, vol. 2018, Article ID 3569139, 6 pages, 2018.

[16] A. Ghorbani and J. Saberi-Nadjafi, "A piecewise-spectral parametric iteration method for solving the nonlinear chaotic Genesio system," Mathematical and Computer Modelling, vol. 54, no. 1-2, pp. 131-139, 2011.

[17] P. Linz, Analytical and Numerical Methods for Volterra Equations, vol. 7 of SIAM Studies in Applied Mathematics, Society for Industrial and Applied Mathematics (SIAM), Philadelphia, Pa, USA, 1985. 
[18] J. A. C. Weideman and S. C. Reddy, "A MATLAB diferentiation matrix suite," ACM Transactions on Mathematical Software, vol. 26, no. 4, pp. 465-519, 2000.

[19] T. Damian, Matrix Based Operatorial Approach to Differential and Integral Problems, Babes-Bolyai University of Cluj-Napoca Romania, Romania, 2011.

[20] R. D. Small, "Population Growth in a Closed System," SIAM Review, vol. 25, no. 1, pp. 93-95, 1983.

[21] K. Maleknejad and M. Tamamgar, "A New Reconstruction of Variational Iteration Method and Its Application to Nonlinear Volterra Integrodifferential Equations," Abstract and Applied Analysis, vol. 2014, pp. 1-6, 2014.

[22] K. Kim and B. Jang, "A novel semi-analytical approach for solving nonlinear Volterra integro-differential equations," Applied Mathematics and Computation, vol. 263, pp. 25-35, 2015. 


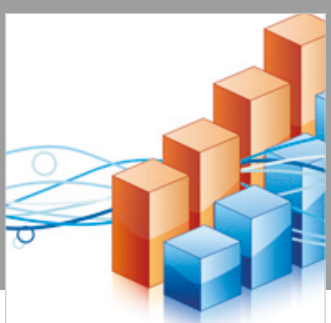

Advances in

Operations Research

\section{-n-m}
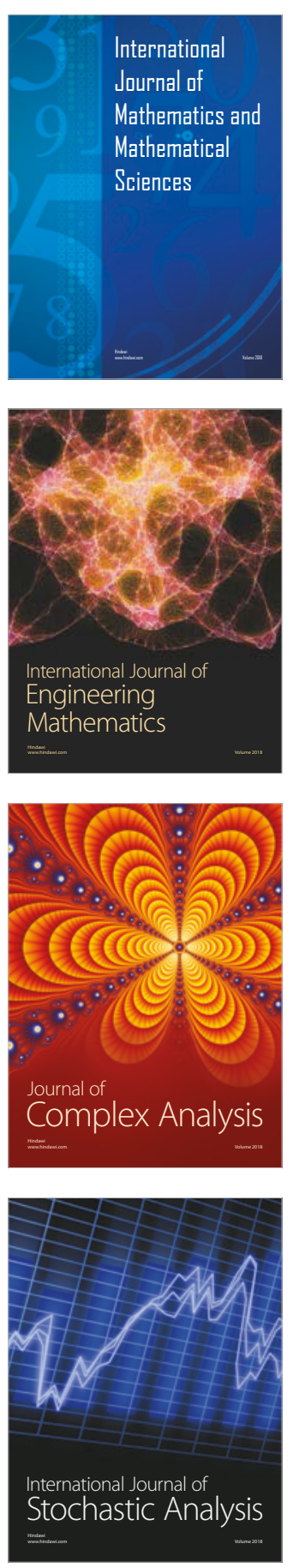
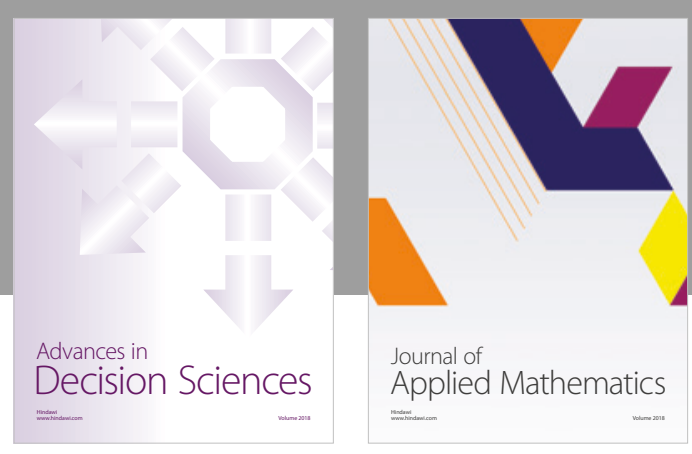

Journal of

Applied Mathematics
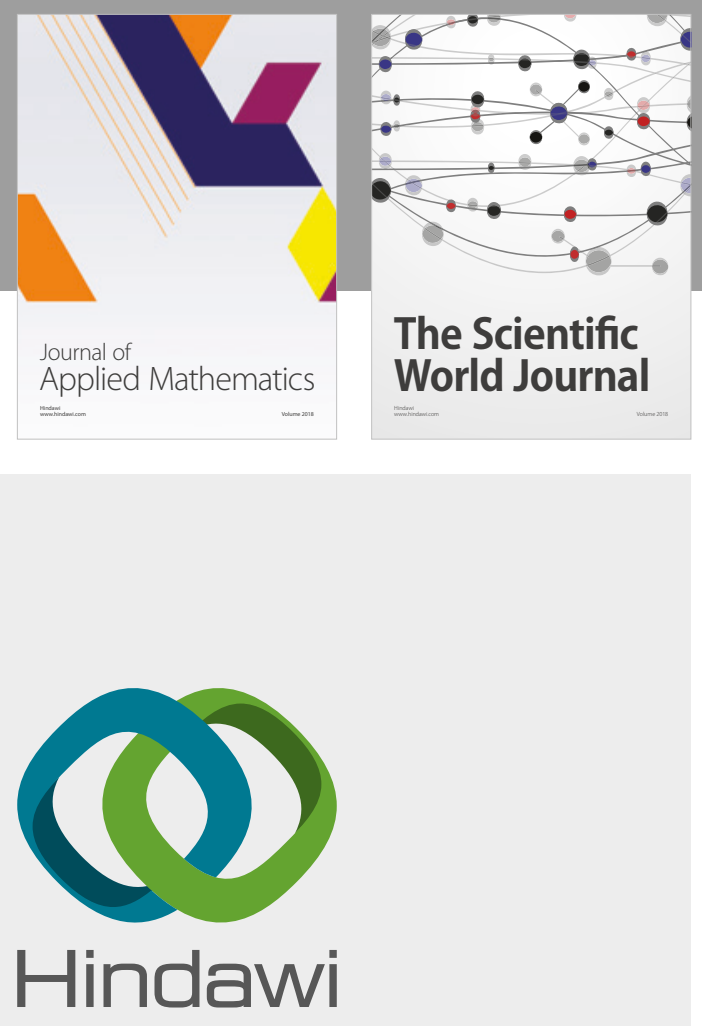

Submit your manuscripts at

www.hindawi.com

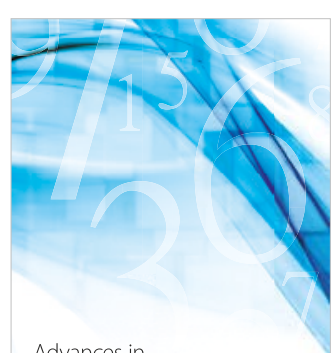

Advances in
Numerical Analysis
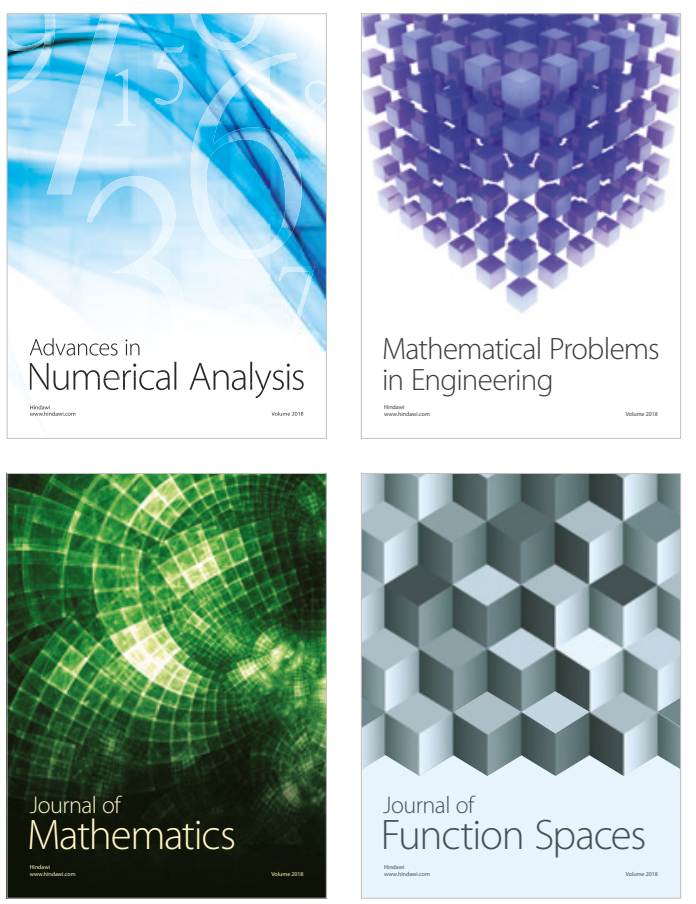

Mathematical Problems in Engineering

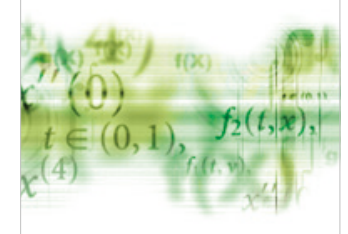

International Journal of

Differential Equations

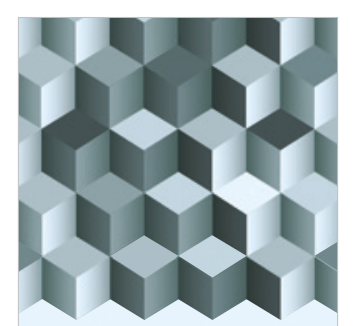

Journal of

Function Spaces

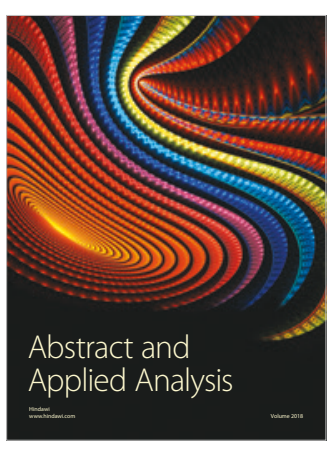

The Scientific

World Journal

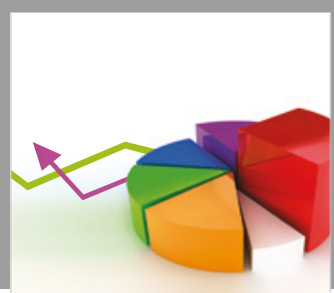

Journal of

Probability and Statistics
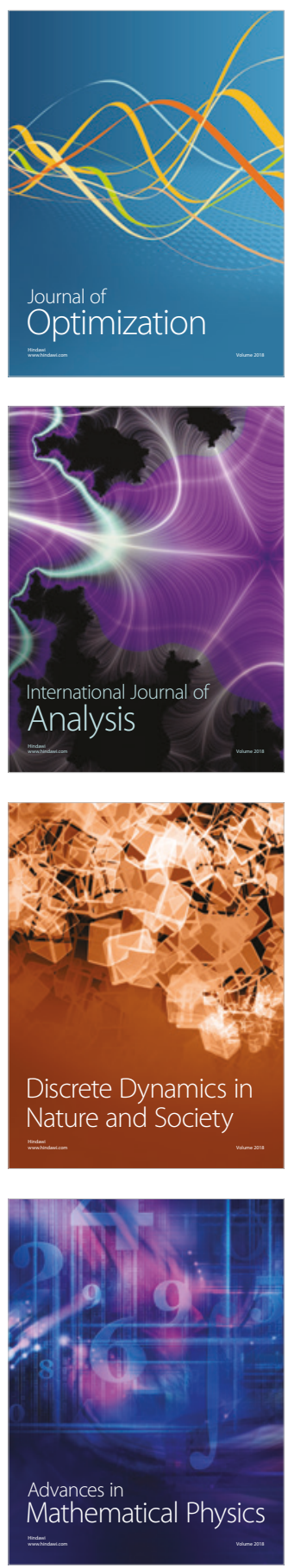